\title{
Non-rigid correction of interleaving artefacts in pelvic MRI
}

\author{
Jason Dowling $^{1 \mathrm{a}}$, Pierrick Bourgeat ${ }^{\mathrm{a}}$, David Raffelt ${ }^{\mathrm{a}}$, Jurgen Fripp ${ }^{\mathrm{a}}$, Peter B Greer ${ }^{\mathrm{b}, \mathrm{c}}$, Jacqueline

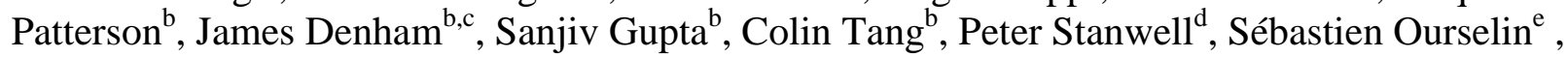 \\ Olivier Salvado ${ }^{\mathrm{a}}$ \\ ${ }^{a}$ Australian e-Health Research Centre, CSIRO ICT Centre, Australia; \\ ${ }^{\mathrm{b}}$ Calvary Mater Newcastle Hospital, Newcastle, Australia \\ ${ }^{\mathrm{c}}$ University of Newcastle, Australia \\ d Brigham \& Women's Hospital, Harvard Medical School, Boston, USA \\ ${ }^{\mathrm{e}}$ Centre for Medical Image Computing, University College London, UK
}

\begin{abstract}
This paper presents a novel method to reduce the effects of interleaving motion artefacts in single-plane MR scanning of the pelvic region without the need for k-space information. Interleaved image (or multipacket) acquisition is frequently used to reduce cross-talk and scanning time during full pelvic MR scans. Patient motion during interleaved acquisition can result in non-linear "staircase" imaging artefacts which are most visible on sagittal and coronal reconstructions. These artefacts can affect the segmentation of organs, registration, and visualization. A fast method has been implemented to replace artefact affected slices in a packet with interpolated slices based on Penney et al (2004) whose method involves the registration of neighbouring slices to obtain correspondences, followed by linear interpolation of voxel intensities along the displacement fields. This interpolation method has been applied to correct motion affected MRI volumes by firstly creating a new volume where every axial slice from the artefact affected packet is removed and replaced with an interpolated slice and then secondly for each of these slices, 2D non-rigid registration is used to register each original axial slice back to its matching interpolated slice. Results show visible improvements in artefacts particularly in sagittal and coronal image reconstructions, and should result in improved intensity based non-rigid registration results between MR scans (for example for atlas based automatic segmentation). Further validation was performed on simulated interleaving artefacts which were applied to an artefact free volume. Results obtained on prostate cancer radiotherapy treatment planning contouring were inconclusive and require further investigation.
\end{abstract}

Keywords: interpolation, non-rigid registration, demons algorithm, MRI artefact correction, prostate radiotherapy

\section{INTRODUCTION}

Interleaved image (or multipacket) acquisition is frequently used to reduce cross-talk and scanning time during full pelvic MR scans and involves acquiring scans non-contiguously (for example slices are scanned in the order $1,17,33,49,2,18,34,50,3, \ldots$, etc ). The slices which are acquired in a single shot (such as $1,17,33,49$ in the previous example) are referred to as a packet (Gedamu et al. [3]).

These scans are often required to identify the prostate border in prostate cancer radiation therapy treatment planning, and could potentially also identify organs at risk for MRI based treatment planning (for example, [2, 5-7]). However patient motion (typically breathing) during interleaved acquisition can result in non-linear "staircase" imaging artefacts which are most visible on sagittal and coronal reconstructions (see Figure 2a.). The method presented in this paper can

\footnotetext{
${ }^{1}$ Corresponding author information: Jason.dowling@csiro.au; http://www.aehrc.com/biomedical_imaging/
} 
significantly reduce these artefacts, which has benefits for the segmentation of organs, statistical atlas generation, and visualization.

This paper presents a new method to reduce the effects of these interleaving artefacts. The method is evaluated on three radiotherapy treatment planning pelvic MRI volumes. As there are noticeable improvements in the visual quality of these volumes, it is hypothesized that the use of corrected planning MR scans will result in an improvement in radiation oncologist manual contouring of organ of interests in treatment planning.

\section{METHOD}

\subsection{Background}

Previous methods to limit patient motion during MRI acquisition have involved pharmacologic sedation, mechanical devices, motion gating or measuring gross patient movement and correcting for its effects. Postprocessing techniques typically require the acquisition of the frequency domain (k-space) data, which is difficult in routine acquisition protocols [1]. Recently Gedamu et al. [3] published a method to reduce the effects of interleaved MRI inter-packet movement correct for brain imaging. Their approach involved extracting all slices from each packet which has been motion affected, use an undefined method to blur those packets in the slice direction to fill the slice gaps (to generate a volume of same size as the original volume); rigidly register the other packets to the motion affected packet, and finally

interpolate (using a Kaiser-Bessel function) a new isotropic volume from the registered packets. Their method is however limited to rigid motion, and needs to be adapted for non rigid deformation associated with breathing motion.

\subsection{Current approach}

The method in this paper involves three main steps (illustrated in Figure 1):

1. First, slices from a packet with interleaving artefacts are removed from the volume. Although our method can be used for a large number of packets, in our MR volumes two packets have been used during acquisition: therefore we can simply remove the even $\left(b_{i . . n}\right)$ slice numbers from the volume, leaving the correctly aligned slices $\left(a_{i . . . n}\right)$.

2. The removed slices $\left(b_{i . . n}\right)$ are then re-created using a registration-based interpolation method similar to that of Penney et al [4] to generate a new set of interpolated slices $\left(i_{i . n}\right)$. The method involves registration between neighbouring slices to obtain correspondences, followed by a linear interpolation of the voxel intensities which are linked by the displacement fields. This provides a much sharper and anatomically correct representation of the missing slice than traditional linear or B-spline interpolation. In our implementation, the free form registration technique used in [4] was replaced by the more computationally efficient demons algorithm $[8,9]$.

3. Finally each of the original, motion affected, slices $b_{i . . n}$ are non-rigidly registered to their corresponding interpolated slices $\left(i_{i . . n}\right)$ using the demons algorithm.

The slices belonging to the packet removed in step 1 are determined subjectively based on contributing slice visual quality (that is, in Figure 1 the packet with the most visible motion artefacts is selected as b and removed from the volume).

The interleave artifact correction method was implemented in C++ under Linux and utilized some classes from the Insight Toolkit ( http://www.itk.org ). 


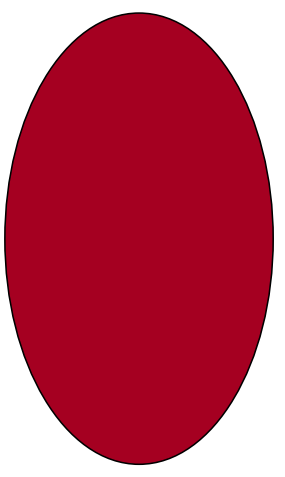

A. Original Object

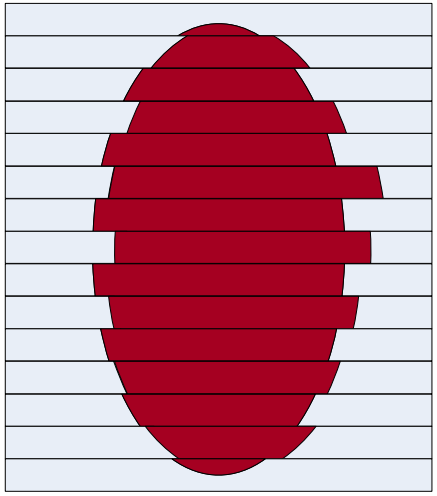

B. Acquired Object Volume

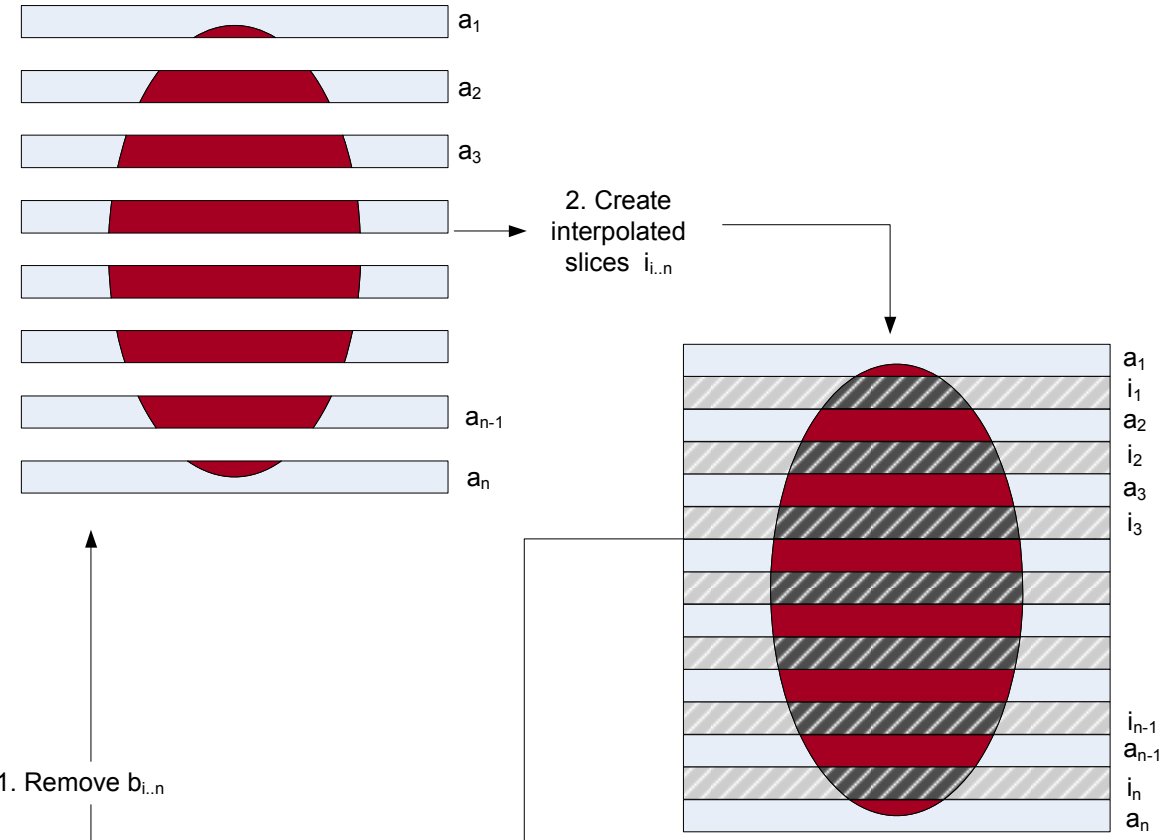

C. Interpolated Volume $b_{n-1}$
$a_{n-1}$
$b_{n}$
$a_{n}$

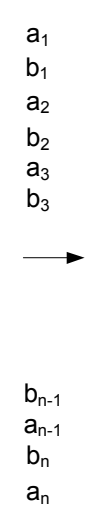

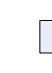

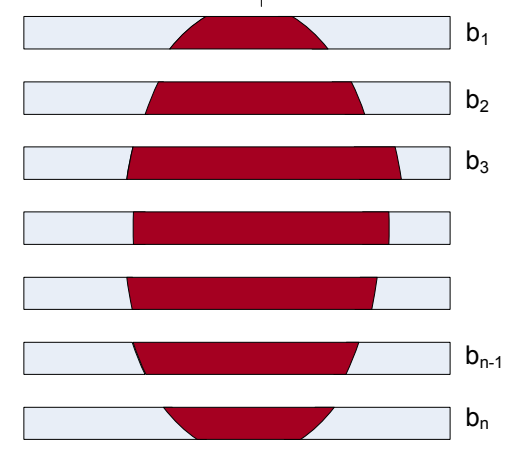

3. Register $b_{i . . n}$ to $i_{i . . n}$

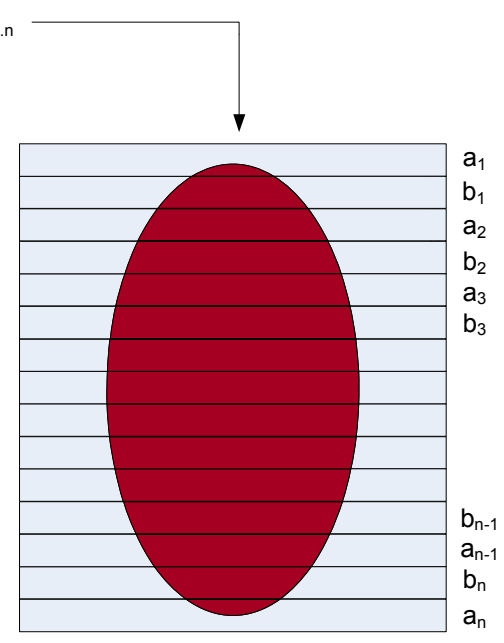

D. Corrected Volume

Fig 1. Diagram showing the main steps involved in correcting the motion artefacts. Object A has been scanned using MRI interleaving (two packets) however breathing motion has resulted in the acquired object volume (B). The steps to correct this volume are: 1 . Remove the motion affected slices from the volume, 2. replace these with new interpolated slices (creating interpolated volume $\mathrm{C}$ ), then 3. use non-rigid registration to warp the original interleaved slices onto the interpolated slices to generate the corrected volume (D). 


\subsection{Validation}

To validate the method, axial proton density-weighted ( $\mathrm{PDw}$ ) whole body pelvic MR images were acquired for prostate cancer radiotherapy treatment planning at Calvary Mater Newcastle Hospital, Australia from a GE Signa 1.5 Tesla scanner using a phased array surface coil with an echo time (TE) of $44.2 \mathrm{~ms}$, repetition time (TR) of $\sim 5000 \mathrm{~ms}$, voxel size $1.56 \times 1.56 \mathrm{~mm}$, and slice thickness $3 \mathrm{~mm}$. Three sagittal reconstructions showing significant motion artefacts are referred to as: A007 (repetition time (TR) =5020, 70 axial slices); C011 (TR=5060, 64 slices) and H003 (TR=5440, 76 slices).

In addition a T2w whole pelvis scan (Figure 3a) without interleaving artefacts (UQ1, dimensions 180 x 256 by 30 axial slices) was acquired for validation using a Siemens Sonata $1.5 \mathrm{~T}$ scanner with an echo time (TE) of 93ms, repetition time (TR) of 5910ms, voxel size 1.56x1.56 mm, and slice thickness $4 \mathrm{~mm}$. To simulate interleaving effects, a cropped set of deformation fields was acquired from the correction of A007. These fields were then inverted and applied to the UQ1 volume (Figure 3b).

\subsection{Evaluation of the interleave correction on manual contouring}

The evaluate the effects of the interleave correction method on radiotherapy planning for prostate cancer, manual segmentations of the prostate, bladder and rectum on the original and interleave-corrected volumes were made by the same radiation oncologist. Manual segmentations were exported from the Phillips Pinnacle treatment planning software (Philips Radiation Oncology Systems, Fitchburg, WI, USA) in RTOG format ( http://www.rtog.org ) and parsed to generate 3D binary segmentation volumes for each organ of interest. Organ mesh curvature and volume were then calculated (using classes of the Visualization Toolkit, http://www.vtk.org ).

\section{RESULTS}

Figure 2(a) shows an original sagittal reconstruction of a PDw MRI acquisition (A007), (b) displays the reconstruction after alternate axial slices were replaced with the interpolated slices. Figure 2(c) shows the final corrected reconstruction.

Sagittal reconstructions of the original, deformed, interleaved and corrected UQ1 volume are shown in Figures 3 a-d.

During prostate cancer radiotherapy treatment there is a need to minimize the dosage applied to the bladder and rectum (to reduce post-treatment complications). Figure 4 shows significant reduction in interleaving artefact around the patient's bladder. These corrected reformations show significant correction of the bladder, rectum and prostate which may have an effect on manual and automatic organ segmentation for radiotherapy planning. The Root Mean Square Error RMSE (and standard deviation) between intensity values in the original, interpolated and corrected slices and the time required to correct each volume are listed in Table 1. These results were obtained on a Dell GX620 (1GB RAM, Pentium 4 dual processor, $3.2 \mathrm{GHz}$ ).

Table 1. RMSE between original and corrected volumes and time required for interleave correction.

\begin{tabular}{|c|c|c|c|c|c|}
\hline Volume & Size $(\mathrm{x}, \mathrm{y}, \mathrm{z})$ & $\begin{array}{l}\text { RMSE original and } \\
\text { interpolated slices }\end{array}$ & $\begin{array}{c}\text { Time to create } \\
\text { interpolated slices }\end{array}$ & $\begin{array}{l}\text { RMSE original and } \\
\text { corrected slices }\end{array}$ & $\begin{array}{c}\text { Time to deform } \\
\text { original slices }\end{array}$ \\
\hline A007 & $256,256,70$ & $34.51(6.34)$ & $13 \mathrm{~m} 21 \mathrm{~s}$ & $25.26(6.19)$ & $14 \mathrm{~m} 56 \mathrm{~s}$ \\
\hline $\mathrm{C} 011$ & $256,256,64$ & $27.22(4.52)$ & $12 \mathrm{~m} \mathrm{20s}$ & $20.29(5.10)$ & $13 \mathrm{~m} \mathrm{37s}$ \\
\hline H003 & $256,256,76$ & $25.43(8.38)$ & $14 \mathrm{~m} \mathrm{43s}$ & $20.41(9.29)$ & $16 \mathrm{~m} \mathrm{13s}$ \\
\hline UQ1 & $180,256,30$ & $34.85(2.75)$ & $3 \mathrm{~m} \mathrm{58s}$ & $34.60(2.62)$ & $4 \mathrm{~m} \mathrm{32s}$ \\
\hline
\end{tabular}




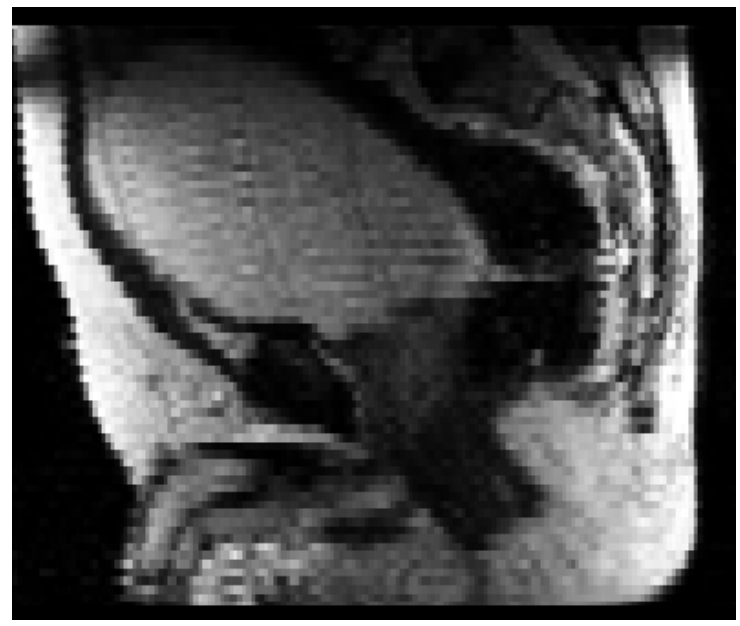

(a)

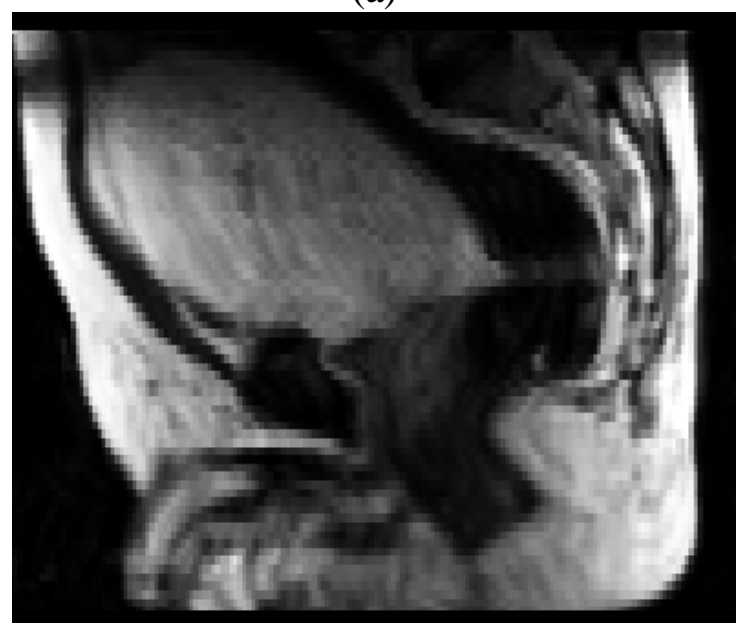

(b)

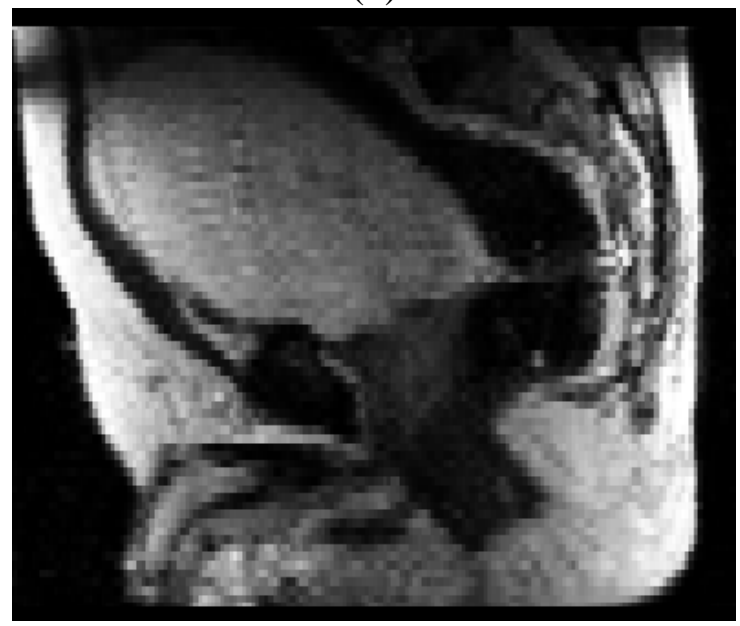

(c)

Fig 2. An original sagittal reconstruction from patient A007 is shown in (a), (b) shows the reconstructed volume after axial slice interpolation, and (c) displays the result of interleave correction (non-rigid registration of original to interpolated axial slices). These reconstructions match items B, C and D in Figure 1. 


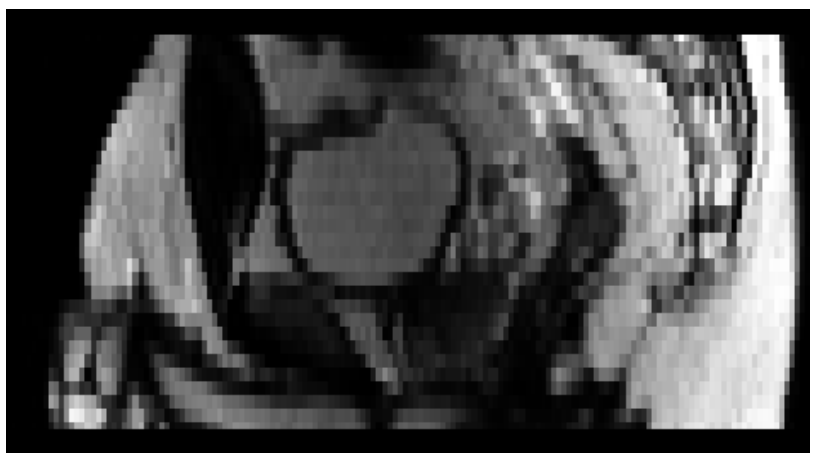

(a) Original volume.

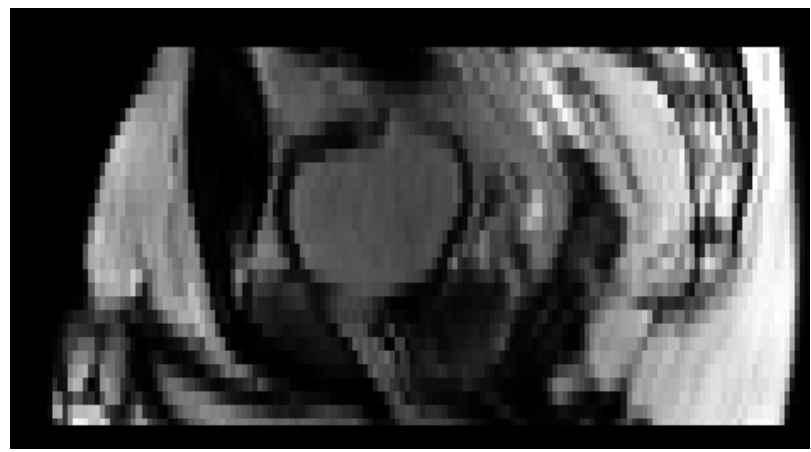

(c) Original after deformed slice interpolation.

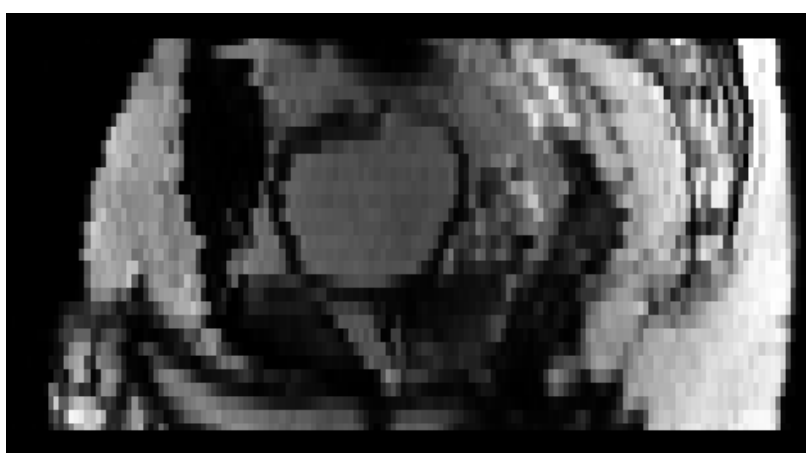

(b) Original deformed with simulated interleaving artefacts.

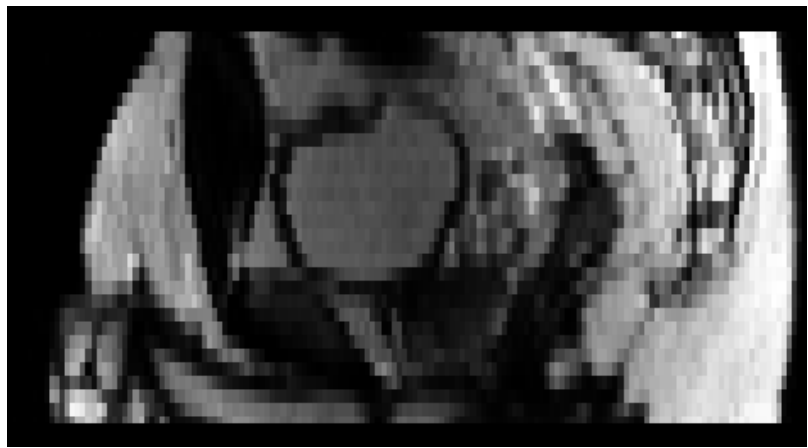

(d) Corrected volume.

Fig 3. Validation results from a pelvic $\mathrm{T} 2 \mathrm{w}$ scan which was acquired without interleaving artefacts (UQ1). A sagittal reconstruction from the original 30 slice volume is shown in (a). This volume has then been deformed with the set of deformation fields from volume A007 resulting in (b). In (c) every second axial slice has been removed and reinterpolated. The final corrected volume, after the deformed axial slices have been non-rigidly registered to the matching interpolated slice is shown in (d).
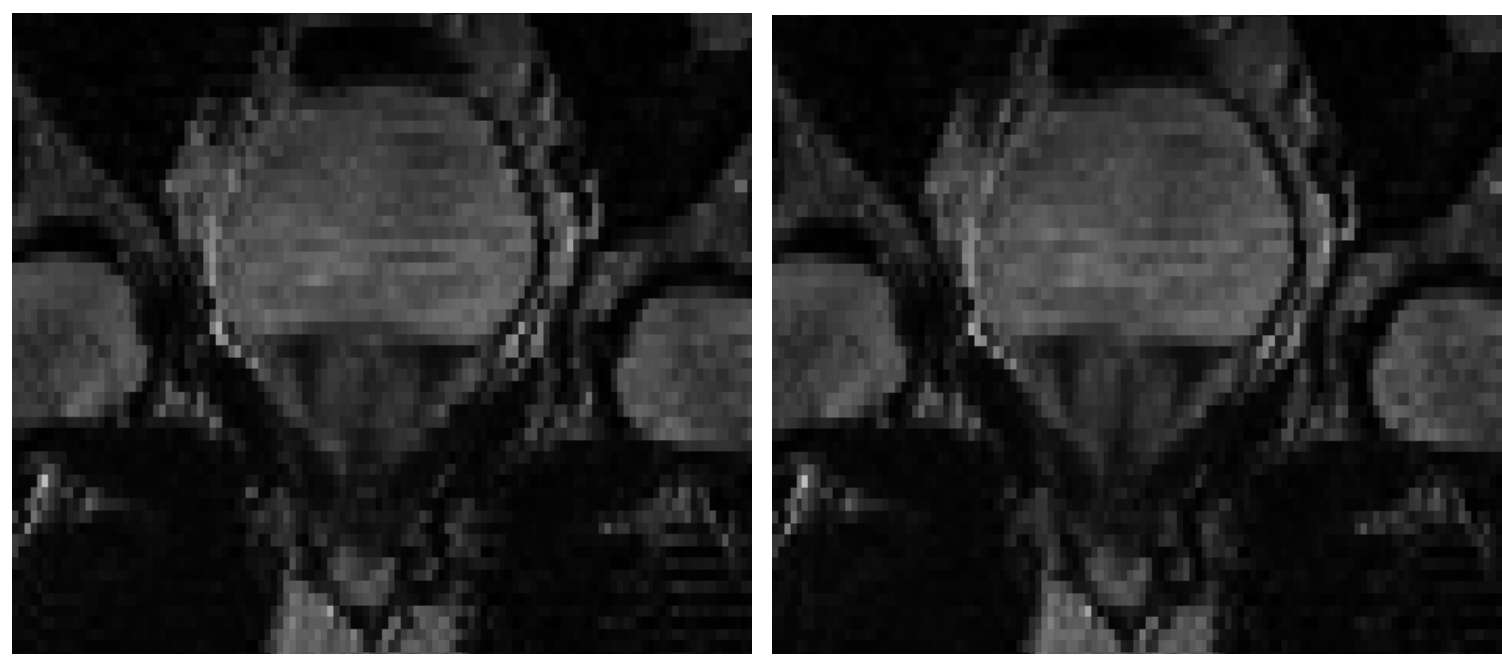

Fig 4. Close up images of a coronal reconstruction view from A007 (a) pre and (b) post correction. Note the clearer bladder boundaries. 

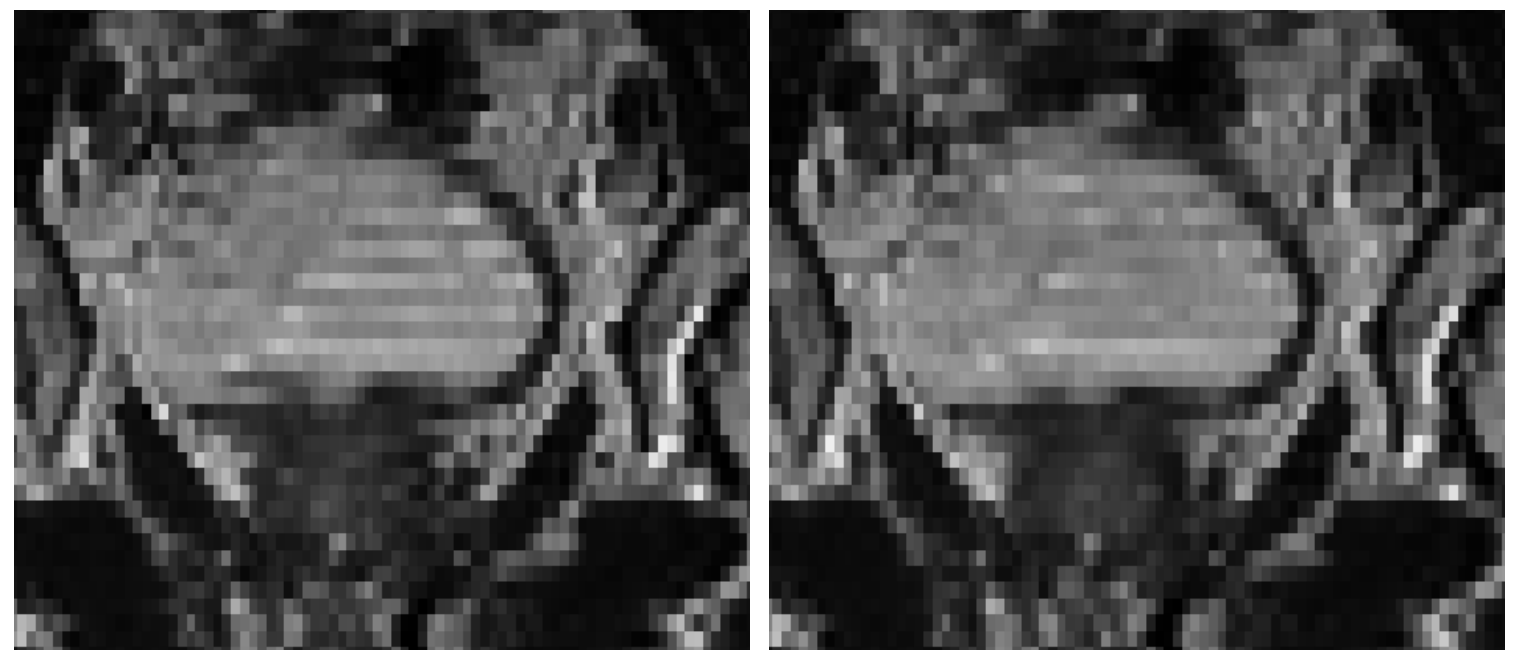

Fig 5. Close up images from a coronal reconstruction view from $\mathrm{H} 003$ (a) pre and post correction (b). The prostate boundaries below the bladder are noticeably improved.
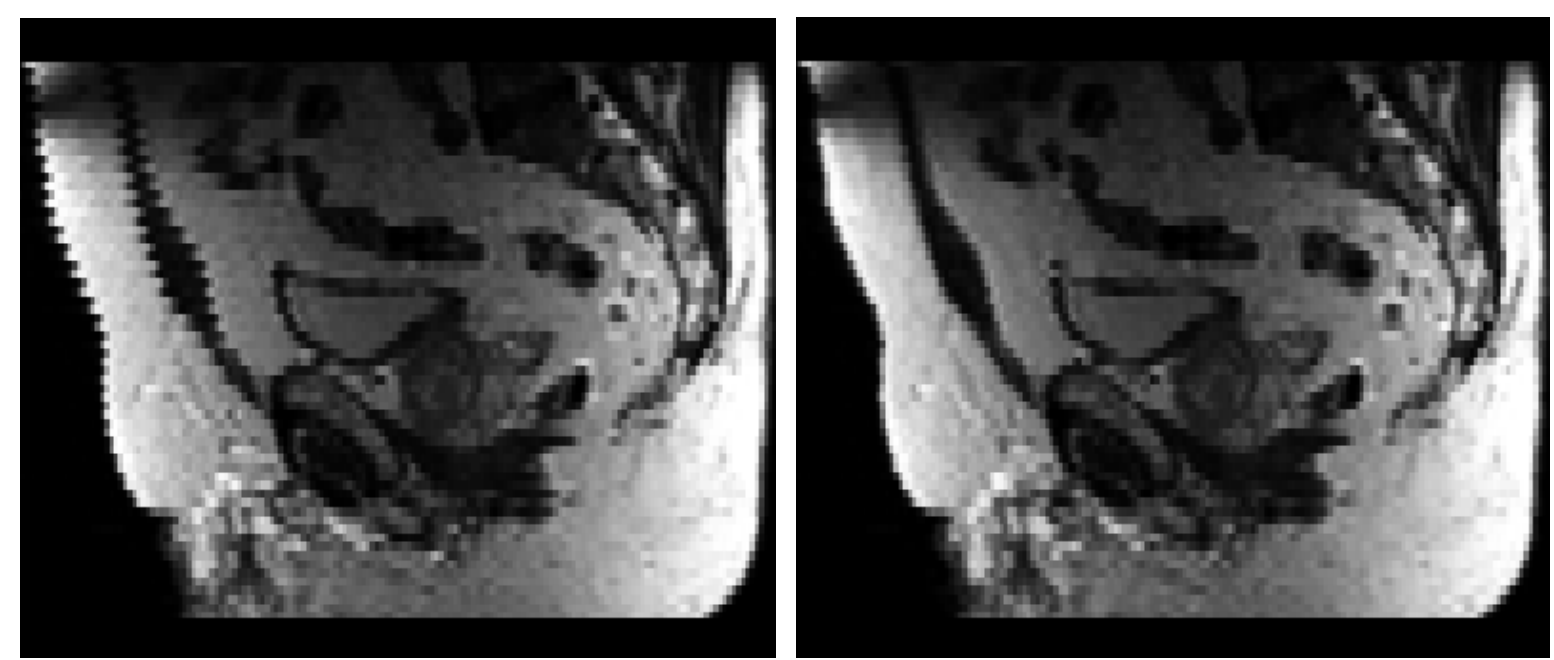

Fig 6. Sagittal reconstruction view from volume C011 (a) pre and post correction (b). The main improvement has occurred in the fat and muscle layers of the patient's stomach.

To investigate the effect of interleave artifact correction on manual contouring, each of the three treatment planning volumes (A007, C011, H003) were corrected and reloaded into the Pinnacle radiation planning system (Phillips). Manual contours were then redrawn on the corrected volume.

As illustrated in Table 2 some differences were found between the organs before and after correction. The volume of the bladder was consistently reduced in the three post-correction volumes (mean volume $295807.5 \mathrm{~mm}^{3} \mathrm{vs} 284469.3 \mathrm{~mm}^{3}$ ). There was a considerable difference in volume between the bladder and rectum for patient C011 before and after correction. The prostate was relatively constant in size between volumes and before and after interleave correction. 
Table 2. Voxel count and volume differences in manual organ segmentation before and after interleave correction.

\begin{tabular}{rrrrrr}
\hline \multirow{2}{*}{$\begin{array}{r}\text { MRI } \\
\text { Volume }\end{array}$} & Organ & \multicolumn{2}{c}{ Number of voxels } & \multicolumn{2}{c}{ Volume $\mathrm{mm}^{3}$} \\
\cline { 3 - 6 } & & Original & Corrected & Original & Corrected \\
\hline \multirow{2}{*}{ A007 } & Bladder & 86851 & 83006 & 636116 & 607954 \\
& Prostate & 4833 & 5083 & 35398 & 37229 \\
& Rectum & 29646 & 31007 & 217134 & 227102 \\
\hline \multirow{2}{*}{ C011 } & Bladder & 8101 & 7788 & 59334 & 57041 \\
& Prostate & 5413 & 5293 & 39646 & 38767 \\
& Rectum & 5381 & 5118 & 39412 & 37485 \\
\hline \multirow{2}{*}{ H003 } & Bladder & 23774 & 23333 & 191973 & 188413 \\
& Prostate & 3353 & 3371 & 27076 & 27221 \\
& Rectum & 21520 & 21620 & 173772 & 174580 \\
\hline
\end{tabular}

As the interleaving artefacts can visibly result in staircase effects around organs (for example, Figure 2), it was expected that the smoothness of manual segmentations could be enhanced in the corrected volumes. Therefore curvature statistics were calculated on each mesh (after processing with Laplacian smoothing) from the three organs of interest. However these results showed mixed differences in organ mesh smoothness (Table 3).

Table 3. Curvature results for manually contoured organs from A007 before and after MRI interleaving correction.

\begin{tabular}{rrrrrr}
\hline \multirow{2}{*}{$\begin{array}{c}\text { MRI } \\
\text { Volume }\end{array}$} & Organ & \multicolumn{2}{c}{ Number of polygons } & \multicolumn{2}{c}{ Mean curvature } \\
\cline { 3 - 6 } & & \multicolumn{1}{c}{ Original } & Corrected & Original & Corrected \\
\hline \multirow{2}{*}{ A007 } & Bladder & 23506 & 21386 & 0.0905 & 0.0811 \\
& Prostate & 3498 & 3628 & 0.1007 & 0.1065 \\
& Rectum & 17300 & 15844 & 0.0990 & 0.0908 \\
\hline \multirow{2}{*}{ C011 } & Bladder & 5098 & 5148 & 0.0878 & 0.1015 \\
& Prostate & 3038 & 2960 & 0.1055 & 0.1027 \\
& Rectum & 4778 & 4722 & 0.1096 & 0.1228 \\
\hline \multirow{2}{*}{ H003 } & Bladder & 10416 & 9768 & 0.0977 & 0.0910 \\
& Prostate & 2474 & 2282 & 0.1101 & 0.0984 \\
& Rectum & 12494 & 11896 & 0.1059 & 0.1127 \\
\hline
\end{tabular}


Organ meshes from manual contours of the bladder, prostate and rectum are shown in Figure 7. As suggested by the curvature results, the rectum for volume A007 has a smoother shape (7a).
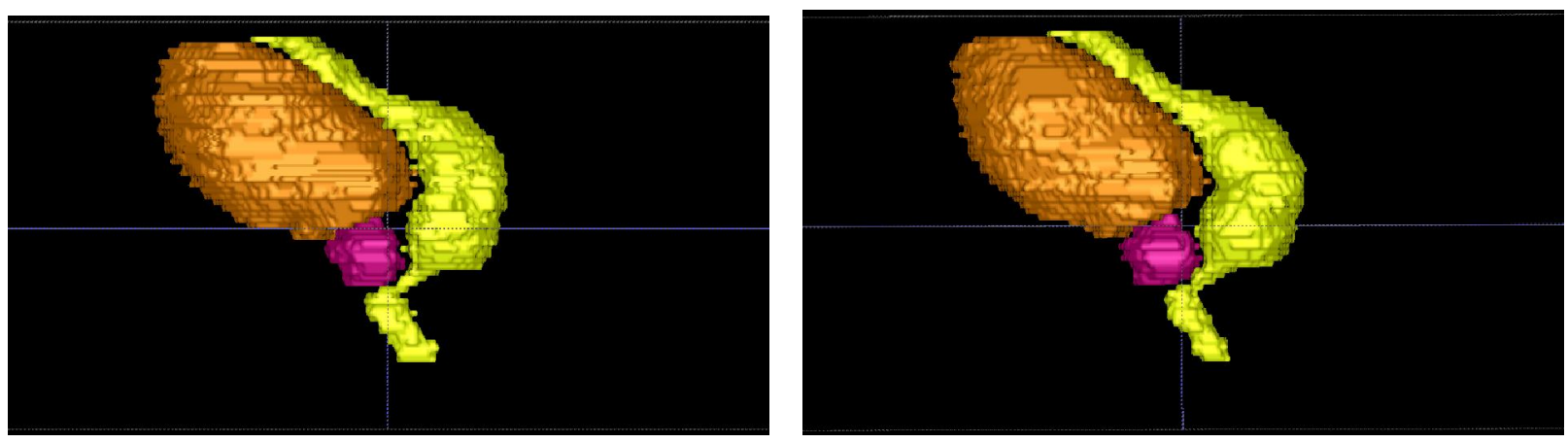

(a) A007 manual segmentation pre and post correction.
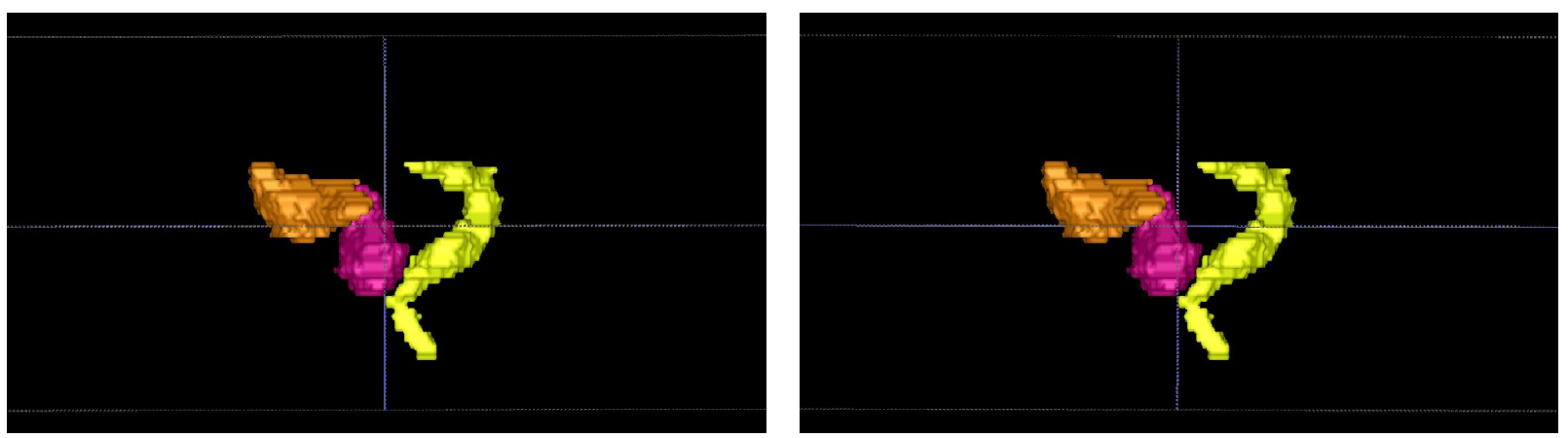

(b) C011 manual segmentation pre and post correction.
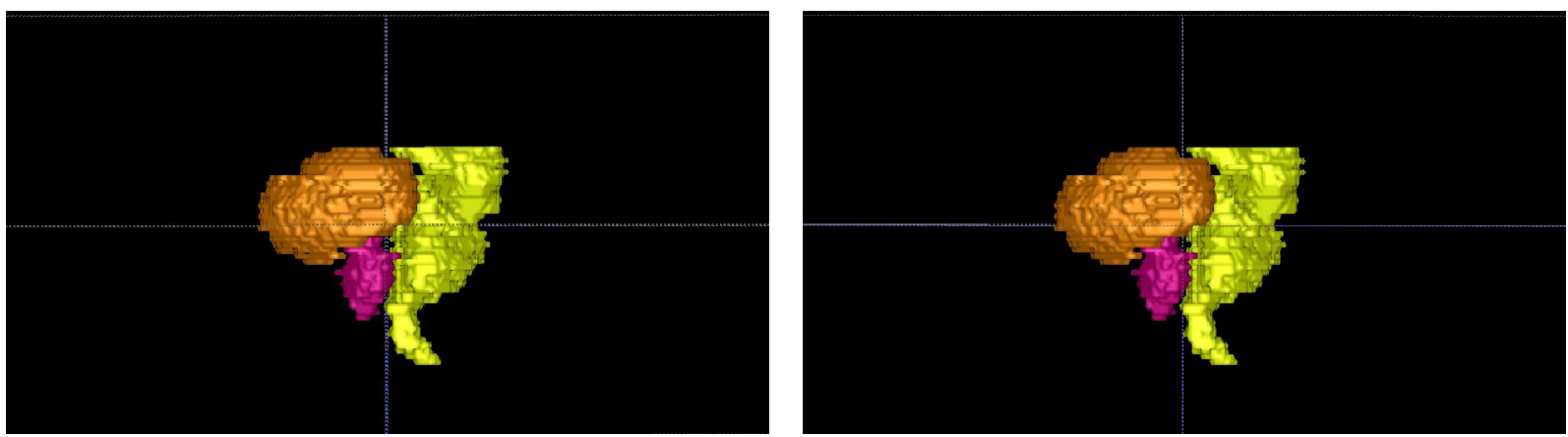

(c) H003 manual segmentation pre and post correction.

Fig 7. Organ meshes generated from the original (left) and corrected (right) manual contours from each of the volumes. The organs are the bladder (orange), rectum (yellow) and prostate (pink). Note the improvement in smoothness for the bladder and rectum in 7(a). 


\section{DISCUSSION}

In this work a fast and novel method for the correction of interleaving artefacts in pelvic MR scans has been developed and application of this method to clinical MR images has been demonstrated. Visible improvements in artefacts are noticeable in sagittal and coronal image reconstructions (for example, Figures 4-6), and should result in improved intensity based non-rigid registration results between MR scans (for example for atlas based automatic segmentation).

The hypothesis that MRI artefact correction would have a beneficial effect on organ delineation in prostate radiotherapy was not supported by the curvature results. Potentially the uncertainties in manual contouring are currently greater than the effect of interleaving, and the $3 \mathrm{~mm}$ slick thickness also introduces inherent stepping artefacts. The volume differences are of interest as these could potentially affect radiotherapy beam design and optimization algorithms which try and minimize the beam size and hence dose to critical organs while maintaining adequate dose to the target volume.

The solution described in this paper adjusts each interleaved slice individually and uses essentially a (fast) 2D approach. However there should be a correlation between each of the slices in each shot, therefore there could be benefits in warping the set of interleaved slices as a $3 \mathrm{D}$ volume to an interpolated volume.

\section{ACKNOWLEDGEMENTS}

This work was partially funded by the Cancer Council NSW, Project Grant RG 07-06. The authors would like to acknowledge the assistance from Ben Brooks from the Centre for Magnetic Resonance, University of Queensland, Australia with the whole pelvis volume for testing.

\section{REFERENCES}

[1] Blumenthal, J.D., Zijdenbos, A., Molloy, E., Giedd, J.N. Motion artifact in magnetic resonance imaging: implications for automated analysis. Neuroimage. 16(1), 89-92, 2002.

[2] Gao, Z., Wilkins, D., Eapen, L., Morash, C., Wassef, Y., Geriga, L.. A study of prostate delineation referenced against a gold standard created from the visible human data. Radiotherapy and Oncology 85(2), 239-246, 2007.

[3] Gedamu, E., Gedamu, A., Arnold, D., Collins, D. MRI inter-packet movement correction for images acquired with non-complementary data. Proceedings International Symposium on Biomedical Imaging (ISBI2008). pp. 416-419, 2008.

[4] Penney, G.P., Schnabel, J.A., Rueckert, D., Viergever, M.A., Niessen, W.J., Registration-based interpolation. IEEE Transactions on Medical Imaging 23 (7), 922-926, 2004.

[5] Rasch, C., Barillot, I., Remeijer, P., Touw, A., van Herk, M., Lebesque, J. V. Defnition of the prostate in CT and MRI: A multi-observer study. International Journal of Radiation Oncology Biology Physics 43(1), 57-66, 1999.

[6] Sannazzari, G. L., Ragona, R., Redda, M. G. R., Giglioli, F. R., Isolato, G., Guarneri, A. CT-MRI image fusion for delineation of volumes in three-dimensional conformal radiation therapy in the treatment of localized prostate cancer. British Journal of Radiology 75 (895), 603-607, 2002.

[7] Steenbakkers, R. J. H. M., Deurloo, K. E. I., Nowak, P. J. C. M., Lebesque, J. V., van Herk, M., Rasch, C. R. N. Reduction of dose delivered to the rectum and bulb of the penis using MRI delineation for radiotherapy of the prostate. International Journal of Radiation Oncology Biology Physics 57 (5), 1269-279, 2003.

[8] Vercauteren, T., Pennec, X., Perchant, A., Ayache, N. Diffeomorphic demons using ITK's finite difference solver hierarchy. The Insight Journal, 2007.

[9] Vercauteren, T., Pennec, X., Perchant, A., Ayache, N. Non-parametric diffeomorphic image registration with the demons algorithm. Proceedings International Conference on Medical Image Computing and Computer Assisted Intervention (MICCAI) Volume 4792, 319-326, 2007. 